\title{
Novel inflammatory biomarkers after long-term left ventricular assist device implantation
}

\author{
Martin Holek ${ }^{1}$, Jiri Kettner ${ }^{1}$, Janka Franekova ${ }^{2}$, Antonin Jabor ${ }^{2}$, Marian Pindak ${ }^{1}$, Hynek \\ Riha $^{1}$, Josef Kautzner ${ }^{1}$, Ivan Netuka ${ }^{1}$, and Jan Pirk ${ }^{1}$ \\ ${ }^{1}$ Institute of Clinical and Experimental medicine \\ ${ }^{2}$ Institute for Clinical and Experimental Medicine Department of Laboratory Methods
}

August 28, 2020

\begin{abstract}
Background and aim of the study: Despite progressive improvement in perioperative care and device technology infectious complications (IC) remain one of the main causes worsening both short-term and long-term prognosis after long-term ventricular assist device (LVAD) implantation. The aim of this study was to assess procalcitonin (PCT) and presepsin (PSEP) dynamics after LVAD implantation and its relationship to IC in the early post-operative period. Methods: A total of 50 consecutive patients indicated to LVAD implantation were included. PCT and PSEP levels were prospectively assessed before surgery and during 30 day follow-up - 1st, 2nd, 14th and 30th post-operative day (POD). Values were compared according to the presence of IC. Results: Both PCT and PSEP levels raised significantly after LVAD implantation. There was no significant difference in PCT or PSEP levels between patients with or without IC during whole follow-up. Patients with acute renal failure (ARF) had significantly higher PCT levels 2 days after surgery and further. ARF increased PSEP levels significantly only 14 days after LVAD implantation. Also subjects with right ventricular assist device (RVAD) had higher PCT and PSEP values. This difference reached the significance only for PCT 14 days after surgery. Conclusions: Our data show that ability of PCT or PSEP to detect infectious complications in patients after LVAD implantation is limited. Their levels more likely correlate with severity of post-operative period in general.
\end{abstract}

Keywords:

Infection, ventricular assist device, inflammatory biomarkers, procalcitonin, presepsin

Introduction:

Long-term ventricular assist device (LVAD) implantation and heart transplantation changed forever therapeutic approach in patients with advanced heart failure and confirmed impact in REMATCH trial ${ }^{1}$. Despite progressive improvement in perioperative care and device technology such as smaller implantable pumps instead of paracorporeal devices infectious complications (IC) remain one of the main causes worsening both short-term and long-term prognosis ${ }^{2}$. Our retrospective analysis of LVAD implantations between 2003 and 2012 identified IC as the most life-threatening complication in postoperative period ${ }^{3}$. Importantly, these complications increase hospitalization $\operatorname{costs}^{4}$.

However, early and accurate diagnosis of IC in LVAD patients is challenging due to serious preoperative status with organ dysfunction and systemic inflammatory response syndrome (SIRS) after surgery caused by cardiopulmonary bypass $(\mathrm{CPB})^{5}$. Among available biomarkers, procalcitonin (PCT) proved superiority over other inflammatory markers such as C-reactive protein after routine non-LVAD cardiac surgery ${ }^{6,7,8,9,10,11}$. But our preliminary prospective data from small cohort showed that ability of PCT to detect IC after LVAD implantation could be limited ${ }^{12}$. In this respect, presepsin (PSEP) is another promising novel biomarker 
for diagnosis of a bacterial infection ${ }^{13}$. The aim of this study was to assess PCT and PSEP dynamics after LVAD implantation and its relationship to IC in the early post-operative period.

\section{Methods:}

This single-center prospective observational study was performed between May 2013 and January 2016. A total of 50 consecutive patients indicated to long-term LVAD implantation (48 HeartMate II VADs, 2 HeartMate 3 VADs; Abbott, Abbott Park, IL, USA) as a bridge to heart transplantation were included (INTERMACS profiles: 2 - 19/50, 3 - 18/50, 4 - 13/50). Exclusion criteria were active infection in 48 hours prior to the surgery, known immune disorder, immunosuppressive drugs in medication and previous short-term LVAD or extracorporeal membrane oxygenation. Preoperative characteristics and baseline values before LVAD implantation are summarized in table 1.

PCT and PSEP levels were assessed before the surgery and during the post-operative period - on the first, second, $14^{\text {th }}$ and $30^{\text {th }}$ post-operative day $(\mathrm{POD}$ ). PCT (reference range $<0.5 \mu \mathrm{g} / \mathrm{L}$; intermediate precision $<2.5 \%$ ) was measured on a Roche Cobas 6000 analyser (Roche Diagnostics, Rotkreuz, Switzerland) with the Elecsys Brahms Procalcitonin kit (cat. no. 05056888; Roche Diagnostics, Mannheim, Germany). PSEP (reference range 60-365 ng/L; total precision $<5 \%$ ) was measured on a Mitsubishi Pathfast analyser using a Pathfast Presepsin kit (cat. no. PF 1201-K; Mitsubishi Chemical Medience Corporation, Tokyo, Japan).

The PCT and PSEP values were compared according to the presence of IC. The definition of the IC was clinically relevant infection (i.e. single positive urine culture without any other signs or symptoms of the infection was excluded) confirmed by at least two physicians in regard to the complete medical chart (sings, symptoms, body temperature, blood samples, cultures, imaging methods etc.).

Values are presented as a median with interquartile range in $\mu \mathrm{g} / \mathrm{L}$ (PCT) or ng/L (PSEP). Statistical analysis using Mann-Whitney test with Bonferroni correction was performed by MedCalc software (version 12.5.0.0) and a $p$ value of 0.05 or less was considered statistically significant. Study protocol has been approved by institutional review board (IRB) and all patients gave written informed consent.

Results:

Five patients (10\%) died during first 30 POD - the reasons were fatal sepsis in one case ( $9^{\text {th }}$ POD), multiorgan dysfunction syndrome (MODS) due to sepsis with right ventricular failure ( $2^{\text {nd }}$ and $22^{\text {nd }}$ POD) in two cases, and MODS without infection $\left(1^{\text {st }}\right.$ and $4^{\text {th }}$ POD) in other two cases.

PCT levels were low before surgery in all patients - actually in a normal range $(0.16,0.10-0.34)$. They raised significantly on $1^{\text {st }}(5.49,2.52-18.42 ; \mathrm{p}<0.001)$ and $2^{\text {nd }}(5.65,2.47-19.53 ; \mathrm{p}<0.001)$ POD. On $30^{\text {th }}$ POD we observed decrease of PCT back to the baseline values (0.09, 0.07-0.19) - figure 1. PSEP levels were mildly elevated before LVAD implantation $(543,340-882)$, raised significantly on $1^{\text {st }}(892,557-1362, \mathrm{p}=0.002)$ and $2^{\text {nd }}(1015,659-1494, \mathrm{p}<0.001)$ POD and decreased on $14^{\text {th }}(838,505-1620)$ and $30^{\text {th }}(566,420-867)$ POD figure 2 .

IC occurred after LVAD implantation in 11 of 50 patients $(22 \%)$. There were 4 cases of sepsis (defined according to the current guidelines) with fatal outcome in one case, pneumonia in 4 cases, urinary infection in one case, and Clostridium difficile colitis in two cases. There was no significant difference in PCT or PSEP levels between patients with or without IC during entire follow-up - see figures 3 and 4 .

Twenty subjects (40\%) had acute renal failure (ARF) defined as injury, failure or loss of kidney function by RIFLE criteria $^{14}$. Seventeen of them needed renal replacement therapy (RRT) initiated between $1^{\text {st }}$ and $7^{\text {th }}$ POD. Patients with ARF had significantly higher PCT levels 2 days after surgery and further (day $2-$ $24.15,4.28-76.7$ vs. $3.3,2.21-9.03, \mathrm{p}=0.045$, day $14-0.68,0.24-2.25$ vs. $0.13,0.09-0.28, \mathrm{p}<0.001$, day 30 - 0.21, 0.12-0.34 vs. 0.08, 0.04-0.12, $\mathrm{p}=0.005)$ - figure 5. ARF increased PSEP levels significantly only 14 days after LVAD implantation $(1926,838-5936$, vs. $688,430-1181, \mathrm{p}=0.005)$ - see figure 6.

Right ventricular assist device (RVAD; CentriMag ${ }^{\mathrm{TM}}$ Circulatory Support System, Abbott, Abbott Park, IL, USA) had to be implanted in 7 patients (14\%) due to right heart failure. Subjects with RVAD had also 
higher PCT and PSEP values. However, this difference reached the significance only for PCT assessed 14 days after surgery $(8.02,0.37-31.95$ vs. $0.17,0.1-0.37, \mathrm{p}=0.018)$ - figures 7 and 8 .

During 1-year follow-up, 10/50 (20\%) patients died. Non-survivors presented with higher levels of both PCP and PSEP before LVAD implantation and during 30-day post-operative period, but this difference did not reach statistical significance (figures 9 and 10).

Discussion:

In the patients with advanced heart failure, LVAD implantation confirmed superiority over optimal medical therapy ${ }^{1}$. LVAD therapy currently represents standard approach in selected patients before heart transplantation (bridge-to-transplantation) as well as in the patients who are not eligible for heart transplantation (destination/permanent therapy).

Despite ongoing technological progress in device construction, e.g. smaller implantable continuous flow pumps and postoperative care improvements including new antibacterial agents or better vital functions monitoring infection still remains life-threating complication after LVAD implantation ${ }^{2,3}$. Altogether, the need for early and accurate diagnosis of this complication followed by appropriate therapy is necessary. PCT has been extensively studied not only in general intensive care settings but also after routine cardiac surgery with promising results in distinguishing infectious and non-infectious SIRS $S^{6,7,8,9,10,11}$. On the other hand, our preliminary prospective data suggested limitation of PCT for diagnosis of the infection after LVAD implantation $^{12}$. In this study we assessed PCT and PSEP (novel inflammatory biomarker) dynamics after LVAD implantation and their relationship to infectious and non-infectious complications.

In our patients both PCT and PSEP levels were significantly higher in first 2 days after surgery compared to preoperative values and then decreased to the baseline level during 30 day follow-up - see figures 1 and 2. While PCT levels were basically normal before LVAD implantation, PSEP levels were already mildly elevated before procedure probably due to non-infectious SIRS. Interestingly, we didn't find any difference in PCT or PSEP kinetics between our subjects with or without infectious complications (figures 3 and 4). Moreover, current papers describing diagnostic utility of PCT after a routine cardiac surgery (CABG, valve surgery or combined procedures) report threshold values for the presence of the infection in the range of 0.47 to $2.47 \mu \mathrm{g} / \mathrm{L}^{6,7,8,9,10,11}$. In our study most of the patients $(41 / 50)$ had at least one PCT value above this range during 30-day post-operative follow-up. And we observed similar situation in PSEP measurements every patient had at least some PSEP level above upper reference limit $365 \mathrm{ng} / \mathrm{L}$ and 42 subjects (84\%) reached cut-off value for sepsis $(729 \mathrm{ng} / \mathrm{L})$. However, our data showed link between other non-infectious complications, such as acute renal failure or right heart failure with additional RVAD implantation, and the levels of inflammatory biomarkers.

The likely explanation is that excessive activation of inflammatory cascade (non-infectious SIRS) in the setting of advance heart failure causes even new inflammatory biomarkers to lose their ability to distinguish infection. While certain pathophysiological processes are unclear, we assume that there are 3 major ways leading to excessive activation of non-infectious SIRS. First, it is well described effect of CPB during cardiac surgery ${ }^{5}$. Second, all the patients were in severe condition with organ failure before LVAD implantation. While in papers describing PCT dynamics after routine cardiac surgery mentioned above most of procedures were elective, all LVAD implantations in our study were urgent or even emergent (INTERMACS profiles: 2 - 19/50, 3 - 18/50, 4 - 13/50). Also preoperative characteristics reflect this fact - $98 \%$ of patients needed intravenous diuretics, $82 \%$ inotropes, $12 \%$ vasopressors, $20 \%$ sildenafil as specific therapy of pulmonary hypertension. Furthermore, mean left ventricle ejection fraction was $25 \%$, only 3 subjects $(6 \%)$ had cardiac index above $2,3 \mathrm{~L} / \mathrm{min} \cdot \mathrm{m}^{2}, 92 \%$ of patients had also right ventricle dysfunction with associated renal insufficiency in 28 subjects $(56 \%)$. We can see this correlation in PSEP values before LVAD implantation, where 34 patients $(68 \%)$ had PSEP levels above upper reference limit $365 \mathrm{ng} / \mathrm{L}$. Even current papers describe the association between PCT and organ dysfunction ${ }^{15}$ or heart failure without any infection ${ }^{16,17}$. And finally, there is a contact of immune cells in the blood with non-physiological surface causing the activation of SIRS ${ }^{18}$. This way is already involved during CPB, but in LVAD pump this process is not short-term 
and limited only to surgery procedure itself. Moreover, with additional RVAD implanted due to right heart failure and/or ARF treated with continuous renal replacement therapy this non-specific activation is much more important. Accordingly, we observed in our patients higher PTC values when ARF occurred (figure 5) and higher PSEP levels 14 days after LVAD implantation, probably caused by renal replacement therapy itself $^{19}$.

In accordance with this hypothesis later decrease in PCT and PSEP levels back to baseline can be explained by improved hemodynamics and organ function with possible immune tolerance to the non-physiological surface.

In addition, we analyzed the correlation between 1-year mortality and the levels of PCT and PSEP dynamics during 30-day post-operative period. We observed higher PCT levels in non-survivors but without statistical significance (in relatively small cohort), and no difference in PSEP values - see figures 9 and 10.

\section{Conclusions :}

Our data showed limited ability of PCT and PSEP to detect infection in the patients after LVAD implantation. Their levels more likely correlate with severity of post-operative period in general. The explanation could be excessive activation of non-infectious SIRS caused by critical perioperative state including organ dysfunction and contact of blood elements with non-physiological surface in ventricle assist device or during renal replacement therapy. In these circumstances the levels of novel inflammatory biomarkers don't discriminate between the activation of inflammatory system due to infection or non-infectious causes.

Conflict of interests:

None.

\section{Author contributions:}

Martin Holek - study design, data collection, analysis/statistics, drafting article

Jiri Kettner - study design, data analysis, critical revision of article

Janka Franekova - study design, laboratory diagnostics, statistics

Antonin Jabor - study design, laboratory diagnostics, statistics

Marian Pindak - study design

Hynek Riha - study design

Josef Kautzner - approval of article

Ivan Netuka - approval of article

Jan Pirk - approval of article

References:

1. Rose EA, Gelijns AC, Moskowitz AJ, et al. Long-term use of a left ventricular assist device for end-stage heart failure. N Engl J Med. 2001;345(20):1435-43.

2. Topkara VK, Kondareddy S, Malik F, et al. Infectious complications in patients with left ventricular assist device: etiology and outcomes in the continuous-flow era. Ann Thorac Surg. 2010;90(4):1270-7.

3. Kettner J, Holek M. HEART FAILURE 2013 POSTER SESSION: CLINICAL (poster no. 672). Wiley Online Library 2014. Available at:http://doi.wiley.com/10.1093/eurjhf/hst009. (abstract)

4. Oz MC, Gelijns AC, Miller L, et al. Left ventricular assist devices as permanent heart failure therapy: the price of progress. Ann Surg. 2003;238(4):577-83. 
5. Laffey JG, Boylan JF, Cheng DC. The systemic inflammatory response to cardiac surgery: implications for the anesthesiologist. Anesthesiology. 2002;97(1):215-52.

6. Simon L, Gauvin F, Amre DK, Saint-louis P, Lacroix J. Serum procalcitonin and C-reactive protein levels as markers of bacterial infection: a systematic review and meta-analysis. Clin Infect Dis. 2004;39(2):206-17.

7. Jebali MA, Hausfater P, Abbes Z, Aouni Z, Riou B, Ferjani M. Assessment of the accuracy of procalcitonin to diagnose postoperative infection after cardiac surgery. Anesthesiology. 2007;107(2):232-8.

8. Prat C, Ricart P, Ruyra X, et al. Serum concentrations of procalcitonin after cardiac surgery. J Card Surg. 2008;23(6):627-32.

9. Samy Kallel, Mohamed Abid, Anouar Jarraya, Mohamed Abdenadher, Emna Mnif, Imed Frikha, Fatma Ayadi, Abdelhamid Karoui. Cinétique et intérêt diagnostique et pronostique de la procalcitonine après chirurgie cardiaque. Annales de Biologie Clinique. 2012;70(5):567-580. doi:10.1684/abc.2012.0745

10. Popov DA, Ovseenko ST, Vostrikova TY. [Procalcitonin as a predictor of bacteremia in postoperative cardiosurgery patients]. Anesteziol Reanimatol. 2014;(2):4-9.

11. Dong Z, Jianxin Z, Haraguchi G, Arai H, Mitaka C. [Procalcitonin for the differential diagnosis of infectious and non-infectious systemic inflammatory response syndrome after cardiac operation]. Zhonghua Wei Zhong Bing Ji Jiu Yi Xue. 2014;26(7):478-9.

12. Kettner J, Holek M, Franekova J, et al. Procalcitonin dynamics after long-term ventricular assist device implantation. Heart, Lung and Circulation, 2017; 26(4): 599-603.

13. Wu J, Hu L, Zhang G et al.: Accuracy of Presepsin in Sepsis Diagnosis: A Systematic Review and Meta-Analysis. PLOS ONE 2015; 10(7), e0133057

14. Bellomo R, Ronco C, Kellum J A et al.: Acute renal failure - definition, outcome measures, animal models, fluid therapy and information technology needs: the Second International Consensus Conference of the Acute Dialysis Quality Initiative (ADQI) Group. Critical care 2004; 8, R204

15. Anand D, Das S, Ray S, Bhargava S, Srivastava LM. Interrelationship between procalcitonin and organ failure in sepsis. Indian J Clin Biochem. 2014;29(1):93-6.

16. Villanueva MP, Mollar A, Palau P, et al. Procalcitonin and long-term prognosis after an admission for acute heart failure. Eur J Intern Med. 2015;26(1):42-8.

17. Loncar G, Tscholl V, Tahirovic E, et al. Should procalcitonin be measured routinely in acute decompensated heart failure?. Biomark Med. 2015;9(7):651-9.

18. Nilsson B, Ekdahl KN, Mollnes TE, Lambris JD. The role of complement in biomaterial-induced inflammation. Mol Immunol. 2007;44(1-3):82-94.

19. Nagata T, Yasuda Y, Ando M et al.: Clinical Impact of Kidney Function on Presepsin Levels. PLOS ONE 2015; 10(6), e0129159 

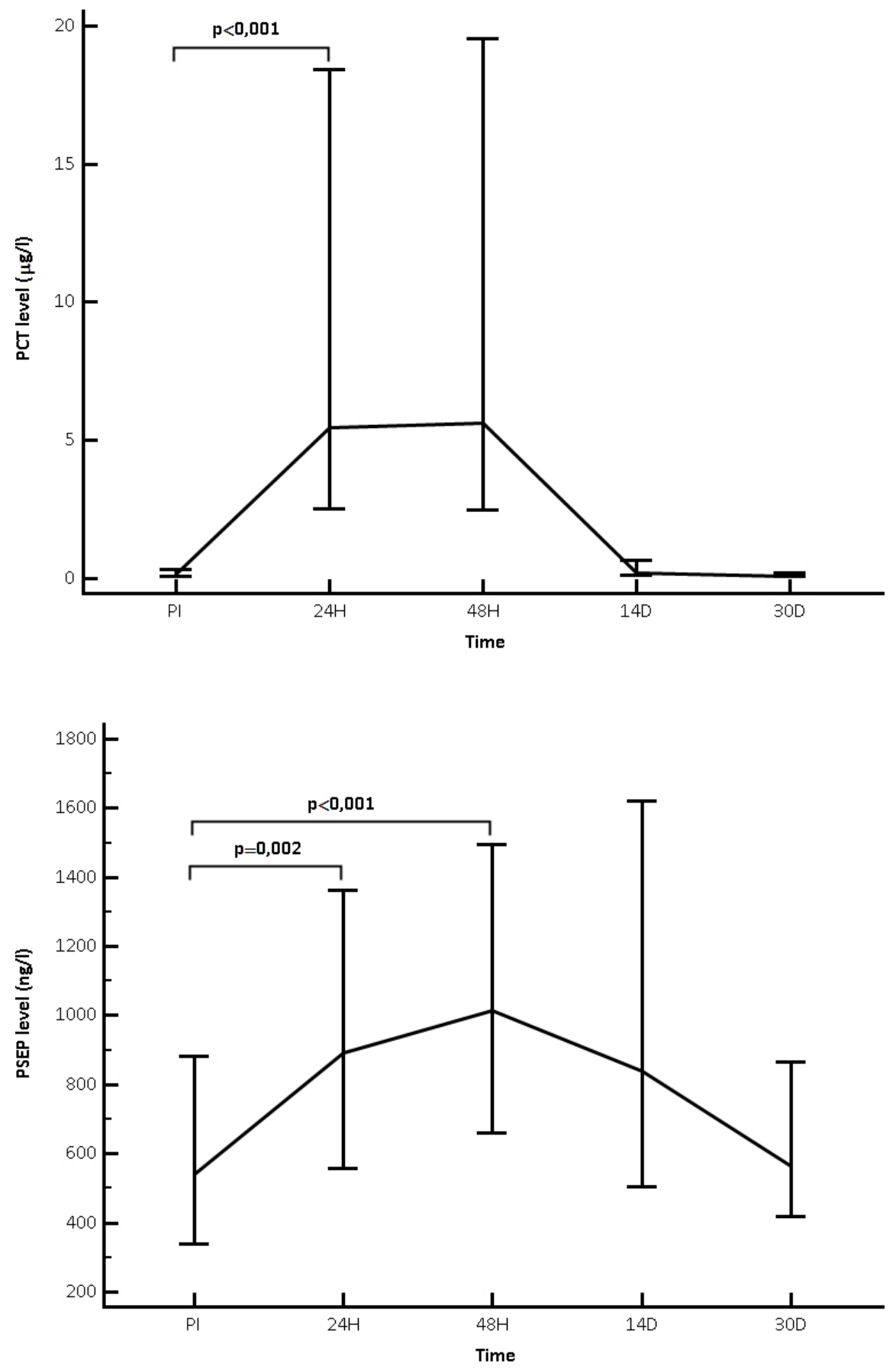

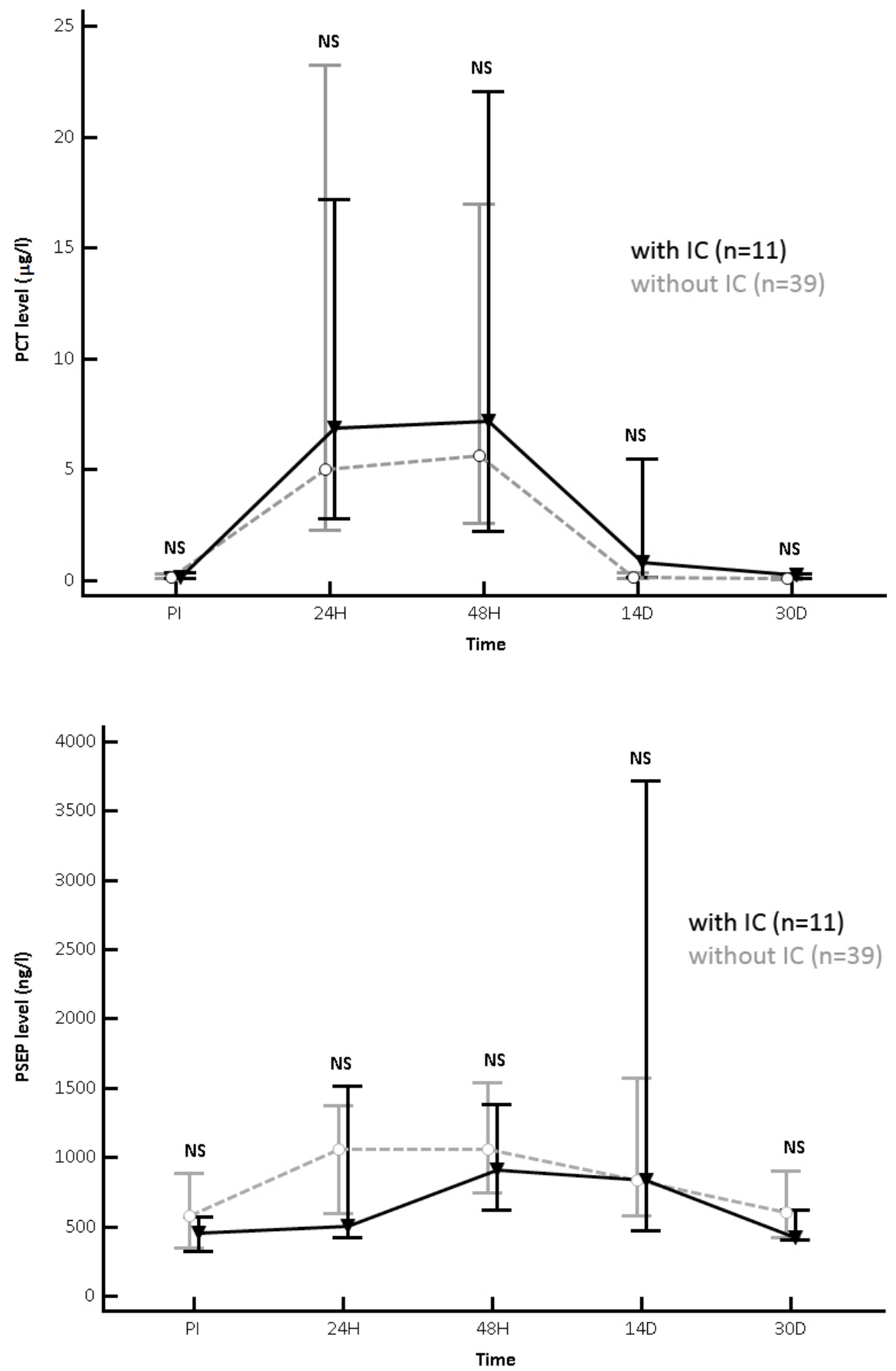

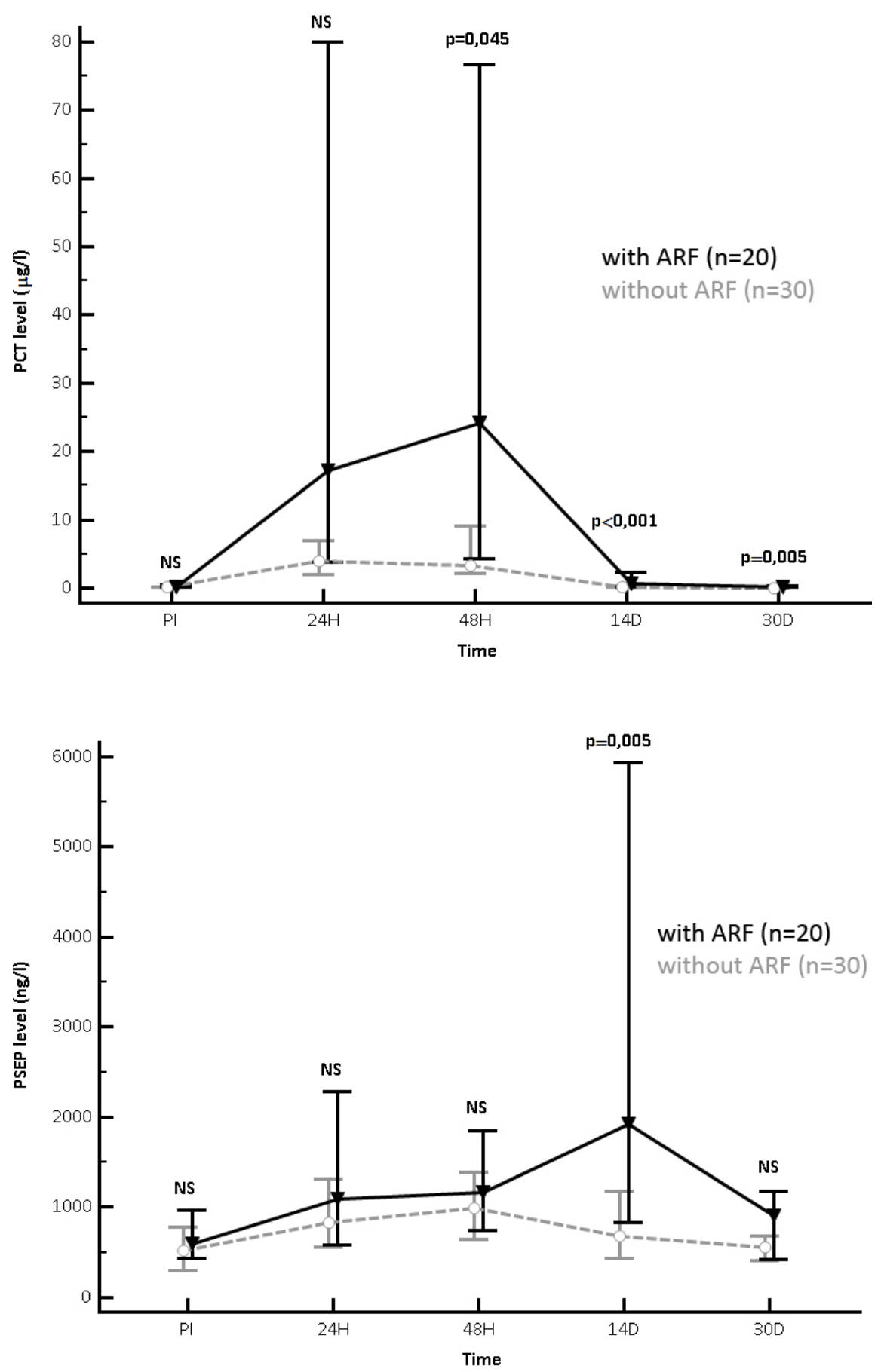

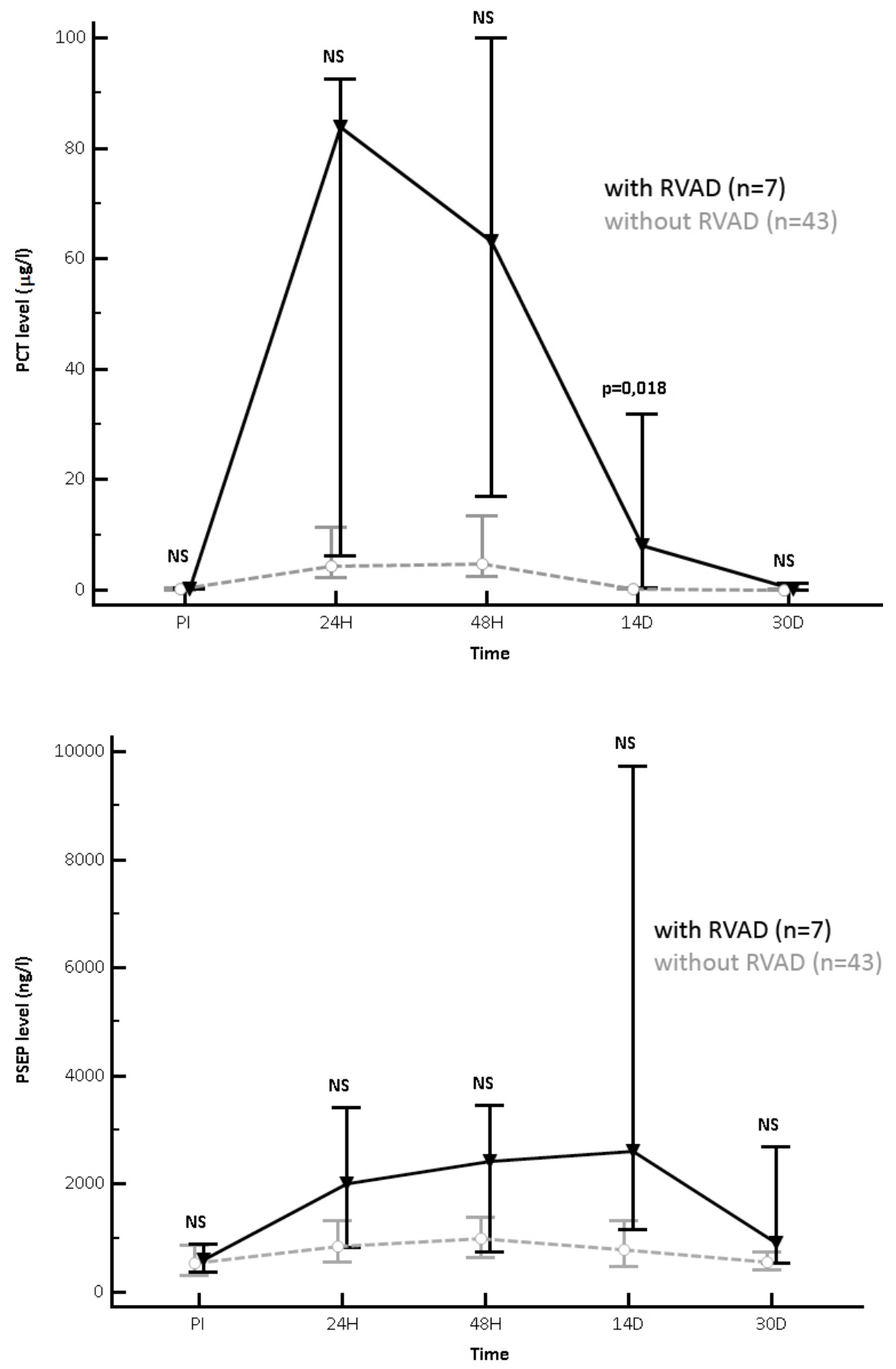

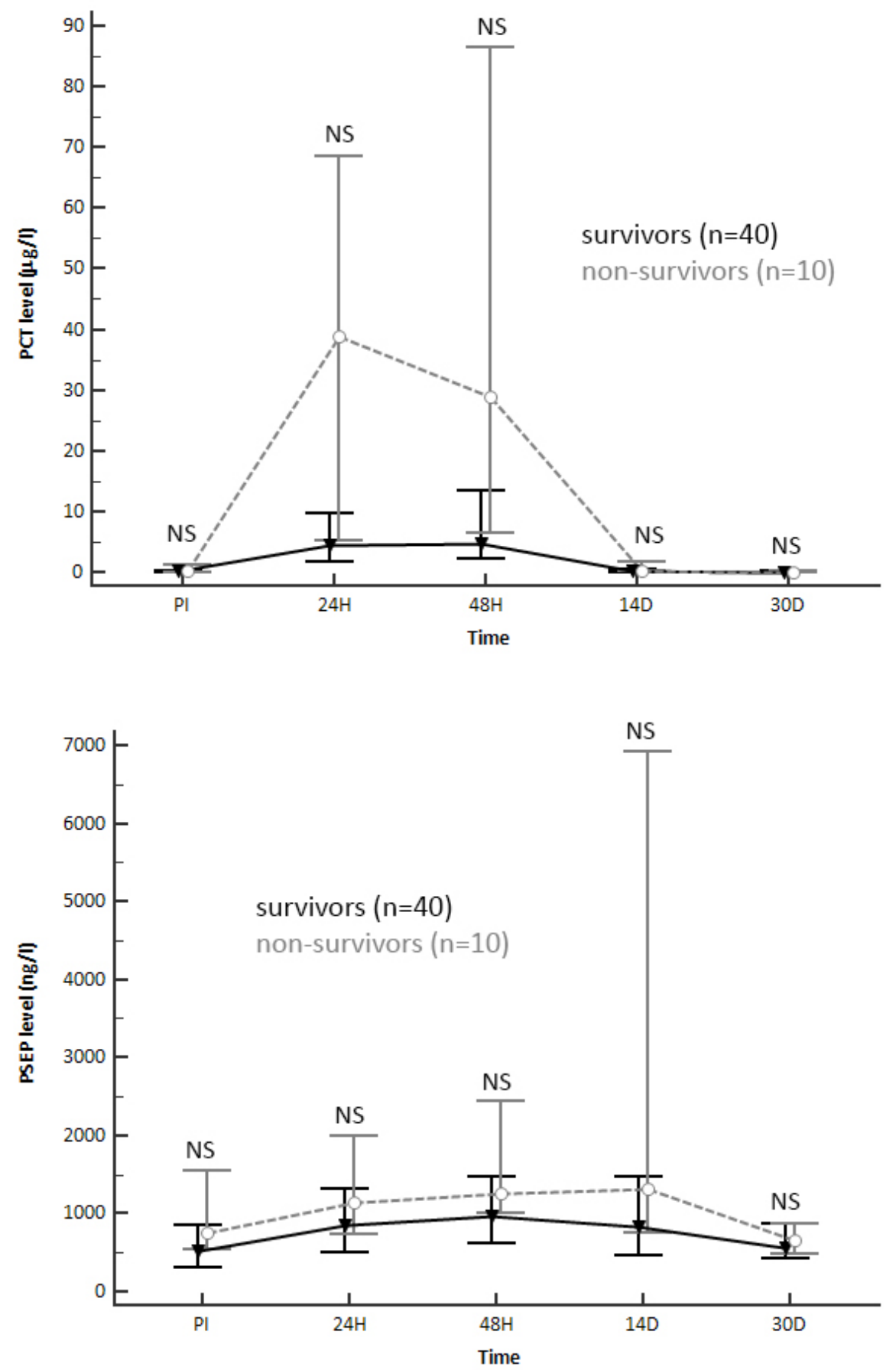

\section{Hosted file}

JCS_tables.docx available at https://authorea.com/users/354302/articles/477904-novelinflammatory-biomarkers-after-long-term-left-ventricular-assist-device-implantation 